\title{
A New Look Care of Mother at Pregnancy \& Lactation: Socio- Cultural Practices in Neglected Population in Kapilvastu District of Nepal
}

\author{
Article by Shashi Kumar Lal Karna \\ School of Public Health, Texila American University, Nepal \\ E-mail: shashikumarlalkarna@gmail.com
}

\begin{abstract}
The study made a new look into existing practice of care of mother during pregnancy and lactation in neglected population predominantly among Muslim, indigenous and lower caste in Kapilvastu district of Nepal. Early marriage, early pregnancy and multiple pregnancies are common socio-cultural practices which is still prevalence in the community.

Women usually go to check pregnancy only for Tetanus injection. The main reason for not visiting check-up is due to family belief that it is 'not necessary'. The husband feels shyness if his wife goes to health facility to see the service providers. The additional foods during pregnancy are not compulsion by the family and women herself. Pregnant women are mostly fed with normal food as usual. Supplementary and more nutritious food during pregnancy are provided especially among economically affluent families. Social belief is that better nutrition during pregnancy will increase baby size which may cause difficulty in delivery and requires operation. Family care and support during pregnancy is low in economically backward communities. Women from poor and Dalit households have high workload during pregnancy.

Home deliveries are more common in compare to institutional deliveries 33.4\%. Delivery at health facility is not necessary', 'not customary' and 'husband or family did not allow to go health facility' as mother in law delivered her many child at home. They try all measures in home; if not succeed then only go to hospital. Women still have their babies at home because of the traditional practice of giving birth at home due to lack of money for transportation facility. Women's low autonomy to decide on maternal health issues could be one of the reasons for low institutional delivery. Unfortunately, women in Kapilvastu district were less aware on danger signs during pregnancy and delivery, and some of the lives threating signs are considered normal. Women who had made sufficient antenatal visit were more likely to deliver at health facility.

People preferred to have maternity services from local traditional practitioners called Chamaini over professional services. The Chamaini helps them in their own home together with family members; they also do massage, and bathing of women and baby. Family members, especially mother in law and relatives also support the women during delivery. The study also revealed that mal-practices in the community are high during delivery. eg, when women find difficulties in removing placenta after birth, there is practice of putting mother's hair in the mouth. People believe that this cause vomiting which results force in uterus and hence placenta is removed.

Heating the place of birth, massage with heated mustard oil to the women during postnatal period is common. Postnatal women are provided to take local made sweet soup called 'Veli' iisoup, which people believe will increase breastmilk and also accelerates uterus constriction. In Tharu culture practices providing to take chilly soup to recently delivered mothers for betterment of mother and child.

Women during postnatal period receive less care and support from the family if the newborn is female child. The care is even less if the women is repeatedly giving birth to female child.

More than50\% of the women are not aware about danger signs during pregnancy. The symptoms such as swelling, vaginal bleeding, dizziness, fever and headache were not considered as threats to the health.
\end{abstract}


Texila International Journal of Public Health

Volume 4, Issue 4, Dec 2016

In home delivery, cord cutting is done by Chamain, until and unless she arrives. The most frequently mentioned cord care by applying naval cream (36.3\%), followed by oil (19.0\%) and Kerosene (17.3\%) and ashes. Around 47\% of the new-borns bath within 24 hours of birth among Kurmi and Dalit ethnicities. In Dalitiii communities, where there is belief that newborns are contaminated with dirty blood are bathed immediately after birth.

Pre-lactating feeds are given in 45\% of the neonates; most common being sweet liquid (60.7\%). There is widespread belief that such sweet foods might be more energetic and child will be able to suck breast milk easily. Other pre-lacteal foods include animal milk and plain water. Pre-lacteal feeding are more prevalent among indigenous (54.3\%), Muslim (46.7\%) and Dalit (44.9\%).

Women herself hardly make decision to seek maternal care by themselves. Husbands and mothers-in-law were the one who have final saying in such decisions. Very low proportion of women alone had final saying in decisions related to whether to have another child (5.0\%), going for ANC visit (9.3\%) and place of delivery of the baby (8.7\%).

\section{Introduction}

Globally 7.2 million children die before their fifth birthday each year, more than $40 \%$ (2.9 million) of them die during their first four weeks of life and 273,500 women die due to maternal cause. Maternal death is the leading cause of death among women of reproductive age in Nepal. It is not only a health disadvantage but also a social disadvantage. Maternal Mortality Rate is 170 per 100,000 live births in Nepal (Annual report 2013). Similarly Neonatal Mortality is 33/1000 live birth. The women of reproductive age do not die from disease but during the normal, life enhancing process of pregnancy; delivery and the puerperium and most of which are avoidable. The pattern of maternal and neonatal health care utilization behaviour is furthermore problematic in Kapilvastu district.

In most of the cultures in rural Nepal, mothers are considered ritually "polluted" until Nwaraniv and are restricted to stay at home, preventing access to care during this critical period. Also, new born bathing immediately after birth and applying oil and turmeric powder to the cord stump are commonly practiced traditions and are known to increase the risk of neonatal infections. These are common practice in Kapilvastu.

\section{Objectives of the study}

The primary objective of the study is

To know the maternal and neonatal health care service utilization behaviour (antenatal, delivery, postnatal and neonatal care services).

To find out the cultural and social norms related to maternal and neonatal health care practices.

\section{Study methodology}

The study design was cross sectional, using both quantitative and qualitative methods. The quantitative part of the study was more concerned with assessing the behaviour pattern related to maternal and neonatal health care utilization, husband's supportive involvement in maternal health, while the qualitative part tried explore the underlying socio-cultural factors associated with maternal and neonatal health behaviour including service utilization.

Study methods (Data collection tools and techniques)

The study methods primarily consisted of questionnaire survey with women having children below one year of age, focus group discussion (FGD) and in-depth interview (IDI).

\section{Focus group discussion (FGDs)}

The FGDs was conducted with mothers/women's groups, decision makers (father-in-law, mother-in-law and husbands) and members of Health Facility Operation Management Committee (HFOMC). A total of 20 FGDs: 16 with members of mother's groups, 4 with mixed groups. The participants of mixed group FGDs included husbands and mother in-law 
of pregnant and recently delivered women, HFOMC members, community leader, general people, Female Community Health Volunteer (FCHV) and teacher.

\section{Semi structured in-depth interview (IDI)}

In-depth interviews were carried out with pregnant women, recently delivered women, family members, decision makers (family members, husbands, Mother In-Law, Father InLaw), selected gate keepers (Traditional birth attendants e.g. Chamain, religious leaders e.g. Moulana, Badgadh, Bhalbata, etc.), health workers from local health facility, traditional medical practitioners (e.g. Bengali doctors,) and community leaders, school teachers and other stakeholders

The qualitative tools included questions related to prevailing socio-cultural practices related to maternal \& neonatal health; service utilization practice; knowledge on danger signs related to pregnancy, delivery, postpartum and new-born

\section{Sample size for qualitative assessment}

In each of the selected Village Development Committee (VDC), 16 FGDs (one FGD per sample VDC) with mothers groups, 4 FGDs with mixed groups and 35 in-depth interviews (with pregnant women, recently delivered women, decision makers, FCHVs, health facility staffs, etc.) were conducted. Sampling for qualitative study was purposive.

\section{Result}

The study revealed that child marriage is largely prevalent in Kapilvastu district. Almost $14 \%$ of the respondents were married before the age of 12 years and around half of the women reported their age of marriage between 12 to 17 years. There is a practice of marriage at first and departure of bride to husbands' house after some time (usually in a couple of years), which is locally called Gauna. Almost 37\% of women had Gauna before the age of 18 years.

Antenatal care (ANC) refers to "care during pregnancy", and includes education, screening and treatment to promote the health and well-being of mother and foetus. Government of Nepal, in alignment with the WHO, recommends all women complete at least four ANC visit.

The proportion of women who received at least one antenatal care during the pregnancy of most recent birth was $89.5 \%$. Around $41 \%$ of the women had four or more ANC visits during their last pregnancy. However, only $36.5 \%$ of women made four ANC visits at 4th, 6th, 8th and 9th months of pregnancy.

Half of the women who did not made ANC visit mentioned that it was not necessary to visit for ANC. They though that in case of normal pregnancy with no pregnancy related problems, it was not needed to go to health facility to see the service providers.

More than $21 \%$ of the women were not counselled on any aspects of pregnancy during ANC visit or they could not remember about counselled issues. Almost $61 \%$ of the women mentioned counselling related to nutrition and iron tablet consumption, followed by TT vaccination during pregnancy (41.5\%), safe delivery preparedness $(25.3 \%)$, danger signs during pregnancy (19.4\%), follow-up visits (13.8\%), family planning (12.5\%), breast-feeding to the baby $(9.0 \%)$ and other (3.8\%).

Around $85 \%$ of the births were protected against neonatal tetanus, while 5\% of the women could not remember whether they had received TT injection or not during last pregnancy. Similarly, 72.1\% took iron and folic acid tablets during pregnancy and/or delivery and 78.9\% of the women were treated by ante-helminthic drug during pregnancy. Iron tablet compliance as recommended is only $11.8 \%$.

Respondents were asked to enlist the danger signs during pregnancy that may require immediate medical support. More than half of the women (53.9\%) did not know any type of danger signs during pregnancy. Women who were aware about danger signs during pregnancy more frequently mentioned haemorrhage (22.3\%), followed by swelling of face and limbs (22.0\%), severe lower abdominal pain (20.7\%), continuous vomiting (17.0\%), high 
Texila International Journal of Public Health

Volume 4, Issue 4, Dec 2016

fever (12.7\%), etc. Other dangers signs such as no weight gain, anaemia, blurred vision, convulsion, high blood pressure and difficulty in breathing were mentioned less frequently.

Women were also asked whether they experienced any danger signs during their last pregnancy. Around $43 \%$ of the women did not experienced any type of danger signs, while $23.2 \%$ mentioned swelling of face and limbs, $19.8 \%$ mentioned continuous vomiting and $16.4 \%$ each mentioned severe lower abdominal pain and haemorrhage. Other danger signs during pregnancy were experienced by relatively less proportion of women.

Women were asked whether they or their family had made any of the four birth preparedness arrangements before the delivery of the most recent birth. About $39 \%$ of the women had made no preparations at all. More than half $(55.7 \%)$ of the women reported saving money, one-fourth (28.5\%) arranged for transportation and only $13.9 \%$ had identified place and person for delivery assistance. Very few women (2.5\%) had identified a person who could donate blood if required. Some of the women also reported that they had arranged food and clothing.

The national health policy of Nepal promotes delivery at a health facility by a skilled birth attendant to ensure appropriate medical attention and hygienic conditions at delivery whereby reducing the complications and serious illness of the mother and baby. Only 33.4\% women delivered their last birth in health facility.

When asked about the reasons behind not delivering at health facility, higher proportion of the respondents reported 'delivery at health facility was not necessary' (36.2\%), followed by 'not customary' (26.3\%), 'husband or family did not allow to go health facility' (22.5\%), 'health facility was too far or there was no transportation available to the facility' (19.2\%), 'high cost related to health facility delivery' (7.5\%), 'child born before reaching facility' (6.6\%) and others.

59\% women did not know any danger signs during labour and child birth. The most commonly mentioned danger signs of labour and childbirth were prolonged labour (labour lasting more than 12 hours) (26.9\%), excessive bleeding (22.0\%), wrong positioning of baby (12.7\%), high fever (8.4\%), retained placenta (placenta not delivered within 30 minutes after delivery of baby) (7.4\%) and convulsions (5.3\%).

Regarding the key danger signs experienced during labour and child birth, more than half (52.6\%) of the women did not experienced any danger signs during their last birth. Prolonged labour, which is one of the top five major causes of maternal mortality and topmost cause of morbidity in low-income countries, was experienced by $17.3 \%$ of the women. Other complications experienced include excessive vaginal bleeding (10.5\%), convulsions (9.6\%), high fever (5.0\%), retained placenta (3.4\%) and wrong positioning of baby (2.8\%).

Women were asked whether they had received their health check-up after delivery. Around $40 \%$ of the women had received at least one PNC after delivery. One-third women (34.1\%) mentioned that they received PNC within 24 hours of delivery. The proportion of women who received PNC during 24 hours to 3 days and 3 to 7 days was $8.0 \%$ each. This shows that PNC visit after 24 hours of delivery is very low. Only 5.3\% women visited for three PNC checksup.

More than 50\% women do not know any danger signs during postnatal period. Among those who were aware of danger signs, mentioned lower abdominal pain, excessive vaginal bleeding, high fever/severe headache and foul smelly discharge from vagina respectively as a danger signs during postnatal period. Other signs mentioned by respondents were weakness (10.8\%), continuous vomiting (7.7\%), convulsions (7.1\%) and redness around nipples (5.6\%).

Regarding the danger signs experienced during postnatal period, $57.0 \%$ of the women did not experienced any danger signs. The danger signs of postnatal period commonly experienced include lower abdominal pain (19.8\%), excessive bleeding (13.3\%), high fever/severe headache (11.8\%) and extreme weakness (10.2\%). Few of the women also mentioned experiencing convulsions (7.4\%), foul discharge from vagina (6.5\%), continuous vomiting (4.3\%) and redness around nipples (4.0\%) 
Women were asked whether they have practiced different type of newborn care immediately after birth. Majority of the newborns (88.5\%) were wrapped with warm clothes immediately after birth, while around two-third of the women (67.2\%) mentioned that their newborns' eye, face and body were cleaned with clean cloth. Around half of the newborn (49.8\%) were initiated breastfeeding immediately after birth. One-fourth (25.15) of the newborns were kept skin-to-skin contact with mother (MayakoAngalo)v.

Women who delivered their youngest baby at home were asked about the umbilical cord care. More than $90 \%$ of the women mentioned that new blade was used to cut the cord, while $4.7 \%$ used old blade or other things. A non-sterile instrument was used to cut the umbilical cord in around $5 \%$ of births

Majority (83.3\%) of the mothers were using some form of application on the cord stump. The most frequently mentioned application were naval cream (36.3\%), followed by oil (19.0\%) and Kerosene (17.3\%).

Women were asked to enlist the danger signs among neonates which need immediate medical attention. Almost half of the women had no knowledge of any and listed none. Among the neonatal danger signs, difficulty in sucking breastmilk (34.1\%), fever (30.3\%), coldness/hypothermia (27.6\%), naval inflammation (19.8\%) and difficulty in breathing (15.8\%) were the most frequent signs mentioned as danger signs. Others include weakness (12.4\%), appearance of rashes (5.9\%) and low birth weight (4.0\%).

Women were also asked whether they had experienced any of the danger signs in their new-borns. Fifty-eight percent of the women did not seen any danger signs in their neonates. The most common danger signs seen by the women in their neonates were fever (23.5\%) followed by difficulty in sucking breastmilk (11.1\%), hypothermia (10.8\%), difficulty in breathing (8.0\%), naval inflammation (7.7\%) and low birth weight (7.4\%). Fewer proportion of women also noticed weakness, rashes, convulsions and yellowness of body.

Only $11.8 \%$ of the women require no permission from husbands or other senior members of the family to go to the local health facility.

The proportion of women who discussed with husbands about the number of children to have was $81.4 \%$. Similarly, $74.6 \%$ of women discussed with husbands on whether to use family planning and $70.0 \%$ discussed about women's health issues.

Qualitative findings of study

There is widespread belief that visiting health facility for ANC is required only if there health problem arises. Household work burden (unavailability of time) and no approval by senior family members were other reasons for not visiting ANC. When asked about the timing of ANC, they thought that whenever there is problem, pregnant women should go for ANC. Some women only go first time just to confirm the pregnancy.

ANC visit among Dalit and Muslim women is low as compared to other ethnicity. Women's status in Dalit and Muslim community is low and they are not supposed to go alone outside house. Women from Muslim communities are not allowed to go alone to visit health facility, which is one of the reasons for low utilization of ANC service among Muslim women. In some communities, people believe that taking iron tablet will cause abdominal discomfort such as gastritis. Some participants also revealed the community beliefs that consumption of iron tablets may cause headache and vomiting and increase the size of baby.

Regarding the feeding during pregnancy, majority of the respondent says that pregnant women are feed with normal food as usual. The Additional foods are not compulsion by the family and women herself. Supplementary and more nutritious food during pregnancy are provided especially among economically affluent families. In some cases, participants also mentioned that pregnant women should not be fed much as it will increase baby size which may cause difficulty in delivery. Fruits and green leafy vegetables are not provided to pregnant women also because of non-availability of such foods on the one hand and poor economic status of the households.

Pregnant women should not be fed much; it will increase baby size which results for difficulty in delivery. If the size of baby is large, women cannot deliver normally and require 
Texila International Journal of Public Health

Volume 4, Issue 4, Dec 2016

operation. Family care and support during pregnancy was found low in economically backward communities. Women from poor and Dalit households have high workload which does not seem decrease during pregnancy. The workload during pregnancy is almost similar to other times. We have to do all the household chores from early in the morning to late night. We do not have time for adequate rest.

Most of the participants reported that families make arrangements for extra money that may require during the time of delivery. Preparation for transportation during emergency and planning for where to deliver were not mentioned in birth preparations in most of the cases. Economically strong and educated families do birth preparedness to some extent, but not by poor households. People believe that pregnancy and having baby is a normal process and there is nothing to prepare.

Most of us do not do any birth preparedness. When any emergency arises we search for help and go to hospital. Most of the respondents in FGDs said that women usually delivery at home. Though people perceive that HF delivery is safer for mother and child, women still have their babies at home because of the traditional practice of giving birth at home as well as lack of money to cover costs related to HF delivery. It is our tradition that we have deliver a child at the place where our mothers in law used to deliver. We go to hospital, only if there is serious problem that the Chamain cannot handle.

People seek help from traditional practitioners more frequently because they placed greater value on them than on medical services especially for normal delivery. They view medical services appropriate only for very severe conditions. Financial barriers and the traditional beliefs are the major reasons for such practice.

We are very much happy to deliver at home because all the family members are available for care and support. In hospital nurses scold if a woman cries during delivery. When women find difficulties in removing placenta after birth, there is practice of keeping hair in the mouth of women. People believe that this may cause vomiting which results force in uterus and hence placenta s removed. For removing of placenta, women are asked to keep hair in their mouth. This will cause vomiting and it results force in uterus and placenta is removed.

In most of the VDCs PNC visit was very low. Women hardly go for PNC after delivery. PNC is practiced only if there is a serious health problem for the mother. One of the ANM in IDI mentioned that PNC service, including the counselling related to postnatal family planning are provided when women come for immunization of the baby. When mother and baby are healthy, there is no need to go for postnatal care to health facility. Even mother go for PNC, there is nothing to do by service provider.

Those who delivered at home were doing unsafe practice such as putting kerosene immersed cloth in vagina to stop the bleeding. The local traditional birth attendants believe that kerosene will help to reduce and stop bleeding. We put kerosene on a cloth, and the cloth is kept in the vagina that will stop the vaginal bleeding. In some VDCs dried ginger is provided to recently delivered women thinking that the ginger will stop bleeding.

Restriction of movement to delivered women outside home for some days is one of the factors which prevent PNC service utilization. The qualitative findings showed that there is a widespread concern that childbirth is a ritually polluting event. Blood coming from delivered women, the cord and placenta, as well the new-born child are considered ritually polluting. Recently delivered women are not considered to worship god for 11 days. During this period, mother and new-born are not allowed to be touched by other people; and they are not allowed to go outside home, are often kept in isolated area inside the house.

Heating the place of birth, heat and mustard oil massage to the women during postnatal period are mentioned frequently by the participants. Oil massage is provided to women even for two times a day during the initial days of delivery. Postnatal women are provided 'Veli'soup. There is widespread belief that the soup of 'Veli' will increase breastmilk and also accelerated uterus constriction. Some of the participants also mentioned providing chilly soup, though less frequently, among Tharu communities. 
In economically backward families (e.g. Dalits), women during their postnatal period are compelled to do normal household work as well as hard work too. In Brahmin/Chhetrivias well as Indigenous ethnicity, better care and support are being provided as compared to other ethnicities. The FGD participants also revealed that women during postnatal period receive less care and support from the family if the new-born is female child. The care is even less if the women is repeatedly giving birth to female child.

Mostly in home delivery, cord cutting is done by local person called as Chamain in the village. Cord cutting is not done until and unless she is there. Most of the FGD participants mentioned using new blade for cord cutting, however, there are still practice of using old blades and other household equipment (e.g. sickle) for cord cutting in remote VDCs of the district. In Dalit ethnicity, cutting the cord is considered ritually polluting event and it is done by the lowest caste women available within the Dalit.

Last time when there was a birth in my neighbour house, the families had to wait for almost 3 hours to the Chamain for cord cutting; even families do not touch the child until Chamain come for curd cutting.

Application to the cord stump continues to be a common practice among the mothers in the area. Traditionally, the most common application used in cord stump is kerosene oil. Now-adays, there is increasing practice of using Navi Malamvii. However, there are many cases in which kerosene oil are used. Their beliefs for using kerosene oil are as an antiseptic. Use of turmeric powder and mustard oil is also frequently mentioned application during FGDs.

New-borns are usually fed pre-lacteal food immediately after birth and the most common pre-lacteal food is sugar and water solution. They think that this might be more energetic and child will be able to suck breast milk easily. Animal milk and plain water are also seen to provide the new-born before initiating breastfeeding.

On the other hand there are significant numbers of participants in the FGDs who believe that it takes time to come breastmilk and breast milk will not come in majority of the women on the first day. FCHV always tell us to put the baby on the breast immediately after delivery but this does not help because the milk is not coming on the first day. My Mother-in-Law provided sugar and water solution to my baby. Most of the women in our community do same. When asked about the knowledge about the position and placement of baby while breastfeeding, women are not aware about how the baby should be placed while breastfeeding. Breast feeding is universal, but in case where breast milk is not sufficient for child only they prefer buffalo milk to feed child.

Previously, new-borns used to be bathed immediately after birth. But now-a-days, newborns bathing is generally done after 24 hours of birth. However, there also exist a number of cases where new-borns are bathed immediately after birth. In Dalit communities, where there is belief that new-borns are contaminated with dirty blood are bathed immediately after birth. One of the Chamain interviewed also supported the statement that new-born should be cleaned and bathed as soon as possible.

Both the mother and new-born, while delivery, are contaminated with dirty blood which should be cleaned. New-borns should not be kept with such dirty things for longer time. I encourage immediate bathing of both the mother and new-born.

As repeatedly mentioned, during home delivery, almost all new-borns are cleaned and wrapped with clothes, but the clothes is not necessarily the new and clean one. Immediately after birth, new-borns are cleaned and put in front of mothers or someone caries them carefully. There is practice that the families prepare for old used clothes (locally called thangna) for the use of new-borns. As a care taking practice oil massage in front of fire is provided to the new born child. Homemade mustard oil is used most frequently. Applying kajalviii (black eye polish), made up of coal to the eyes of the new-born is also found universal practice. Women mentioned protection from evil spirit as the reason for applying kajal.

Discussion was conducted to assess the level of knowledge and experience of women with regard to the danger signs during pregnancy, delivery, postnatal period and the neonates. The 
Texila International Journal of Public Health

Volume 4, Issue 4, Dec 2016

findings showed that most of the women are unaware about such type of danger signs. Few of the participants mentioned some danger signs during pregnancy such as back pain, lower abdominal pain, vaginal bleeding, swelling of face and limbs, vomiting, and dizziness. Haemorrhage, lower abdominal pain and severe headache with fever are mentioned as danger signs in all cases (pregnancy, delivery and postnatal). However, many of the women in FGDs perceived them as normal. Women mentioned that they occur when women get pregnant and that they all disappear after delivery. Some of the women mentioned lying by the fire and take rest when such symptoms arise.

I had morning sickness, dizziness, vomiting, back pain, and leg swelling; I did not do anything as these are normal during pregnancy said one the participants of FGD. Women also lacked neonatal danger signs. Among those who mentioned neonatal danger signs, difficulty in sucking breastmilk, coldness and difficulty in breathing are cited by some of the participants.

People do not consider some of the danger signs as serious issue requiring immediate medical attention. Furthermore, there is widespread belief that a women should only go to health facility if complication arises. Some participants also mentioned that it took households a long time to make a decision to seek care. Even families perceive that they need immediate medical help, there are other barriers associated in making decisions such as lack of money, difficulties in arranging transportation and others.

\section{Discussion}

The issue of maternal and neonatalcare practices in Kapilvastu district in context to 21st century is alarming situation. The national policy of safe motherhood is not complying with sustainable development goal. So, reviewing national policy must be discussed further based on ground reality for strengthening of health services in the district mainly institutional delivery and demand generation through awareness in the community to change the malpractice behavior at home level and proper planning of birth preparedness.

Similarly champion can motivate community to change in sociocultural practices especially regarding early child marriage, early pregnancy and multiple pregnancy. Now time has come to discuss on improvement of Maternal and neonatal health through multisector approach and local sociocultural accepted behavior change communication.

The political parties leader, religious leader, ward citizen forum, civil society, journalist, women groups, microcredit group, cooperative group water users group, forest users group, father in law group, father group, mother in-law group, teacher, student, clubs, NGOs, CBOs all community micro component and community representative should take lead role in social development part. It is said that without community participation, community people involvement in decision making, there is no chance to change the socio culture practices. So it is debatable for further discussion in the community to change the life of mother and children in Kapilvastu.

\section{Conclusion}

Overall, maternal and neonatal care in Kapilvastu district is quite poor. Maternal health care with regard to utilization of health services seems to deteriorate from pregnancy to delivery to the postnatal period. Higher proportion of women made at least one ANC visit, but the subsequent ANC visit is low and birth preparedness practice is low.

Most of the deliveries took place at home, attendants at delivery were mostly unskilled local birth attendants (Chamain), cord cutting practices were still unhygienic, pre-lacteal feeding exists, bathing immediately after birth continues and unhealthy beliefs and practices of breast feeding practices prevail. These all demand for the need of obvious intervention program.

Existing socio-cultural beliefs related to maternal and neonatal care negatively affected the health status of mother and new-born. Poverty and education level of the mothers and families are the hindering factors including negative cultural practices. Lack of access to basic 
maternal healthcare, poverty, illiteracy, women's low status in the society, shortage of health care professional and underutilization of currently available services are other major challenges to improving maternal health in Kapilvastu district.

For the prevailing practices, intervention should be focused towards the selective approach rather than prevailing blanket approach i.e. those factions of the society (especially Dalits and Muslims) which are devoid of relevant knowledge and practice like suppressed and unreached communities should be prioritized first.

As counselling services provided by the health workers were of poor quality and inadequate, the prevailing unhealthy practices in the area should be discussed with the health care providers and communication strategies through inter personal education during antenatal visits, home visits, community discussion and mass media should be launched. Unhealthy practices of maternal and new-born care should also be discussed with family members (husbands and mothers-in-law), relatives, Chamain and other key players in the society so that they take special action in preventing these harmful practices. There is an urgent need to educate mothers or responsible family members especially mothers in-law.

It is concluded that mothers in the study area had inadequate knowledge and carrying out some harmful practices. Behavioural change communication package designed according to the felt need of the community has favourable impact on the knowledge and safe practices of mothers for neonatal care. Improving health facilities, mother's nutrition, women's position in the society such as freedom of movement, and integrating Traditional Birth Attendants into local health services can play a vital role.

Women and family members in Kapilvastu district strongly value pregnancy, childbirth and new-born care traditions and associated cultural beliefs and they profoundly shape women's views of safety and risk during pregnancy and childbirth. To improve maternal and neonatal health status if Kapilvastu district, service providers must genuinely partner with local women inclusive of their cultural beliefs, and provide locally based primary maternity care.

Health service should make women, family members and communities feel culturally safe and culturally respected within their spiritual traditions of birth BCC package should be designed focusing on changing the adverse behaviour of women, mother-in-law, family members and Chamain. The information in the package should contained self-designed pamphlets, posters and visual cards containing simple messages in local languages (Awadhi). Culturally appropriate images should be designed based on community and subject expert feedback as well as a pilot field study. Rather than distributing such aids to women and communities, health workers, FCHVs and community mobilizers should use them while counselling and discussing with women and family members.

The BCC package should include danger signs during pregnancy, delivery postnatal and neonatal period; general pregnancy care, rest and nutrition during pregnancy and lactating period; importance of iron tablet consumption, institutional delivery and PNC; neonatal care practice (care of naval cord, initiation of early breastfeeding, proper breastfeeding practice, bathing after 24 hours, Kangaroo mother care), etc. Alternative strategies for promoting maternal and neonatal health such as home visits by service providers, interactions within women, family members and communities by using participatory BCC materials may be more effective. 
Texila International Journal of Public Health

Volume 4, Issue 4, Dec 2016

\section{Figures and table}

Education status of female vs male:

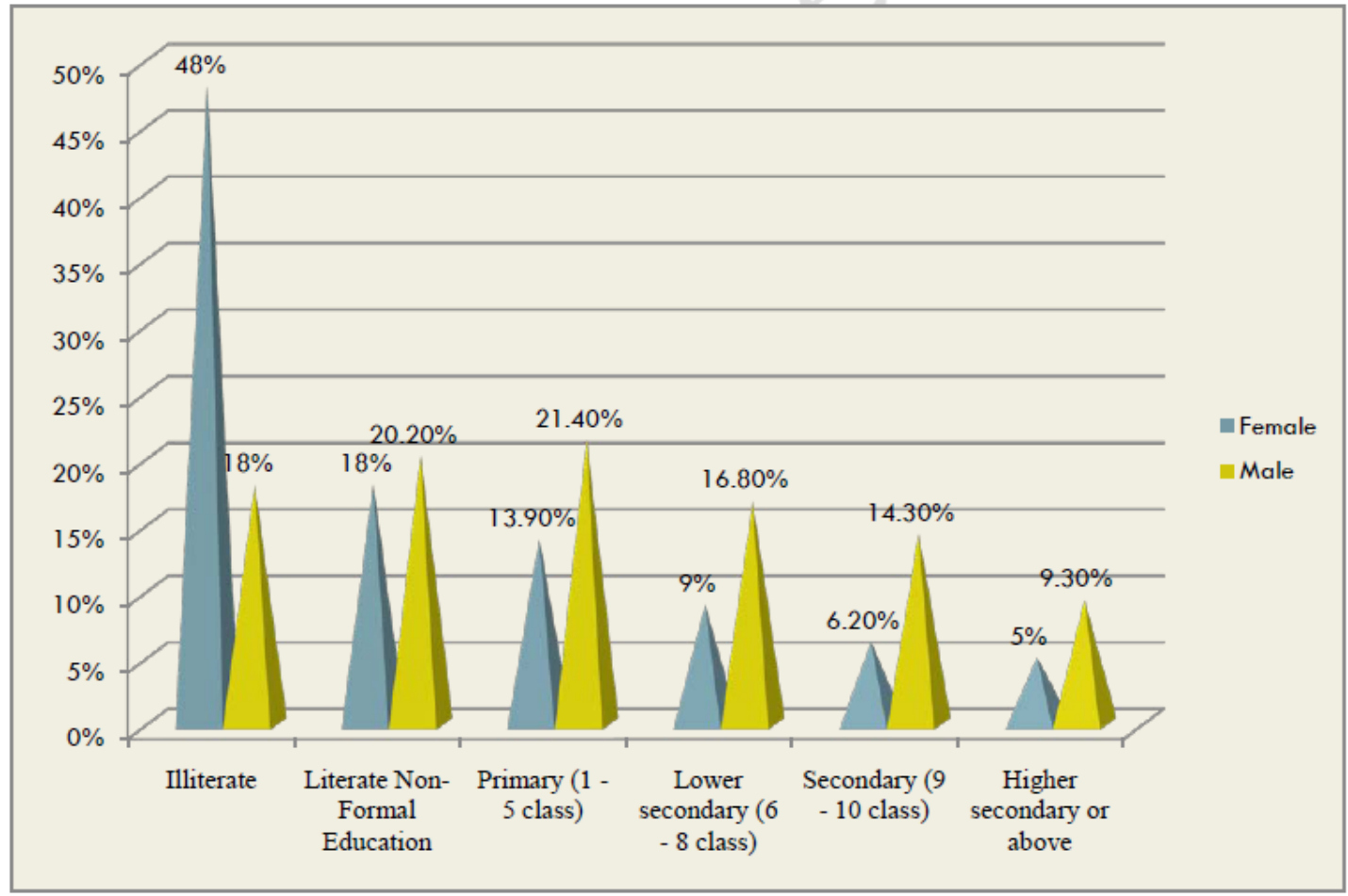

Age of marriage vs first Pregnancy:

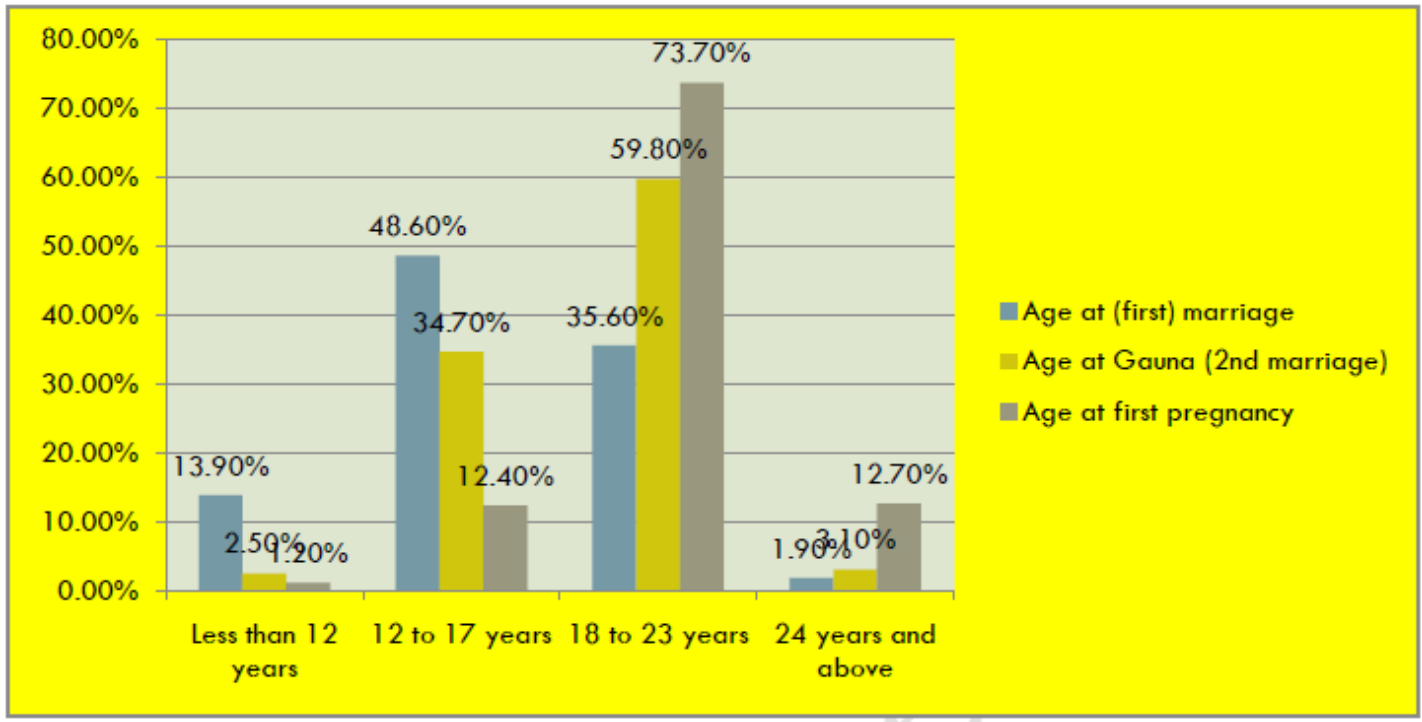


Texila International Journal of Public Health

Volume 4, Issue 4, Dec 2016

ANC service utilization
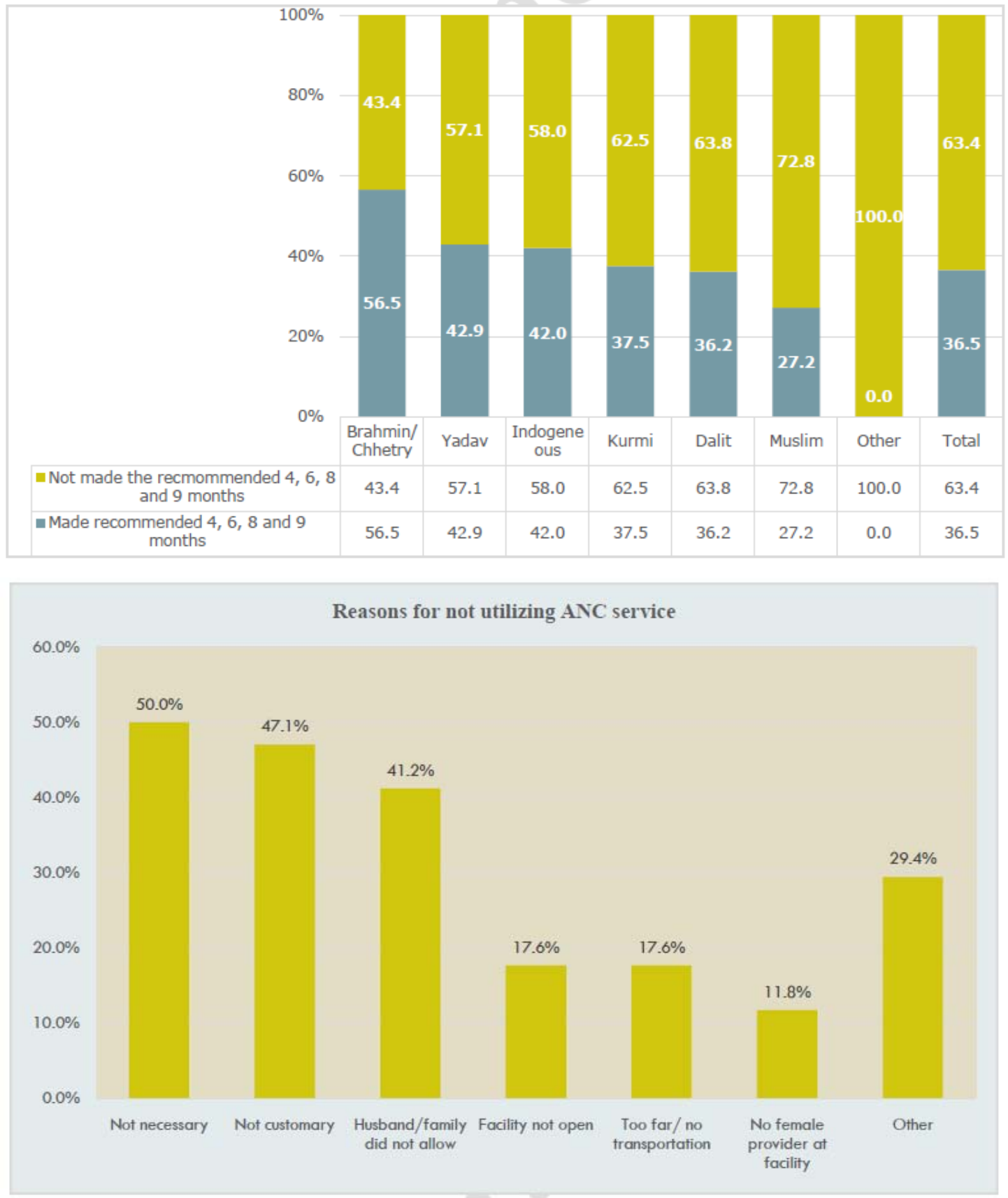
Texila International Journal of Public Health

Volume 4, Issue 4, Dec 2016
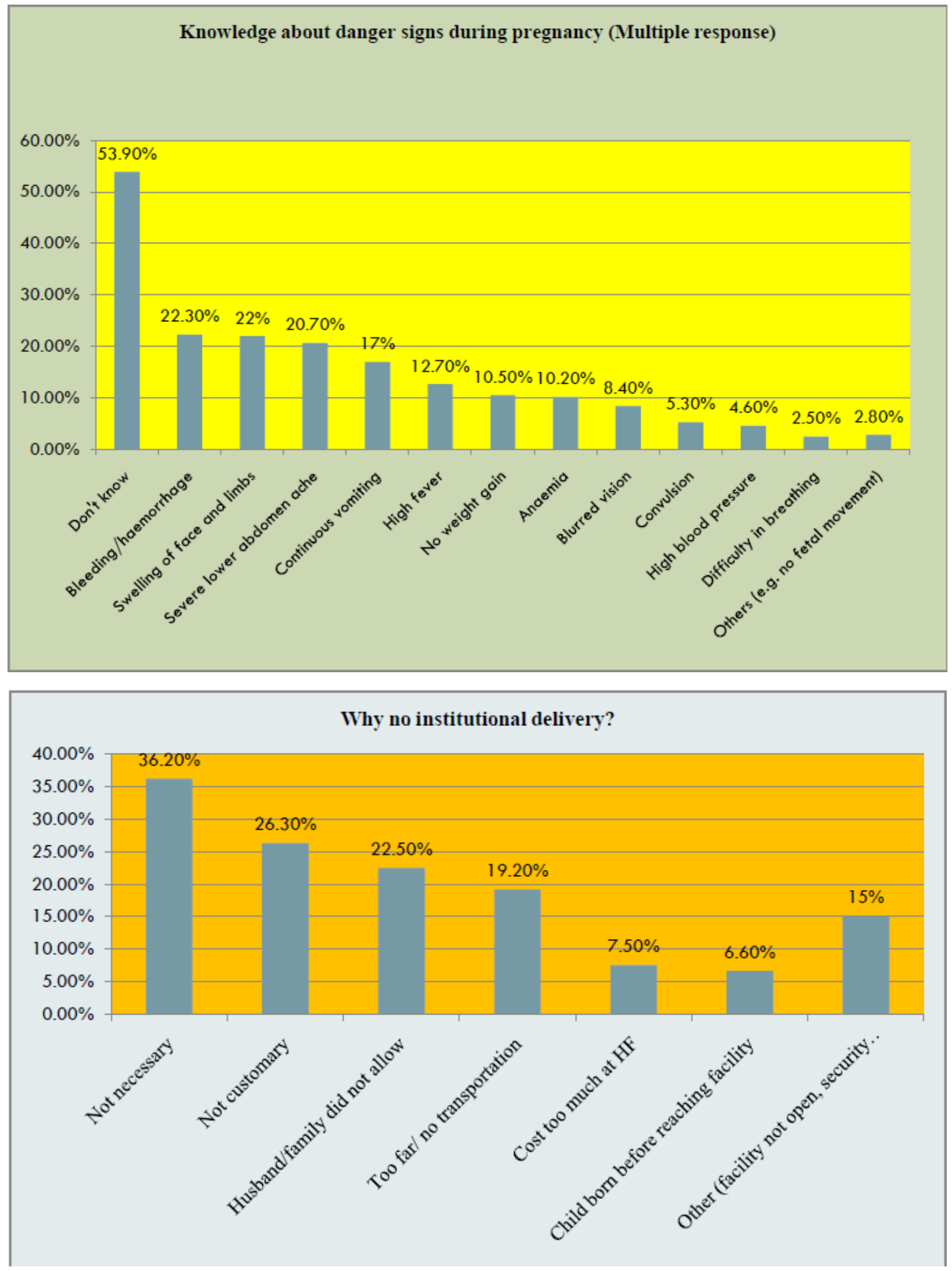
Texila International Journal of Public Health

Volume 4, Issue 4, Dec 2016

Neonatal care after birth
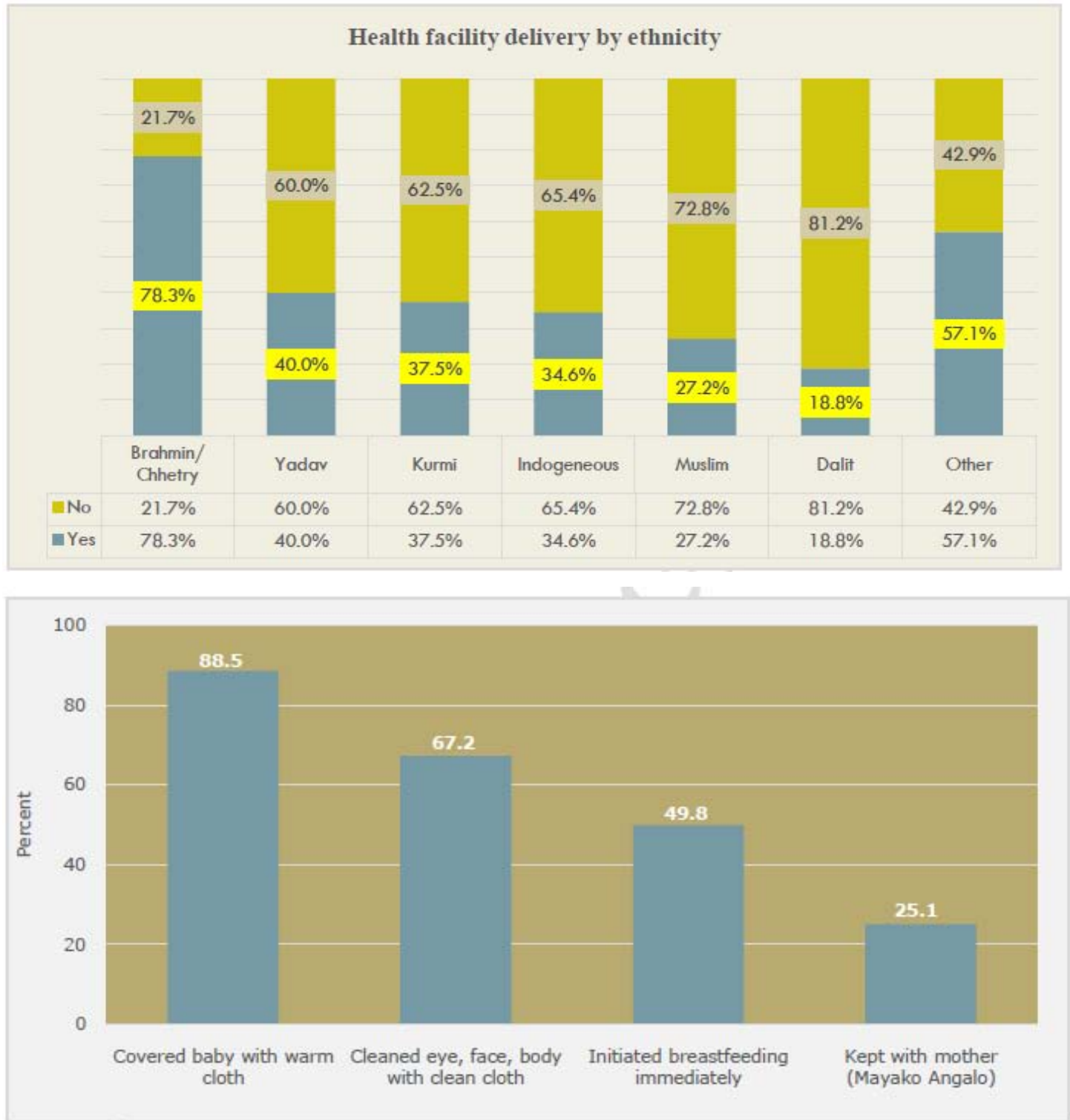

What was used to cut the cord? (Among Home delivery)
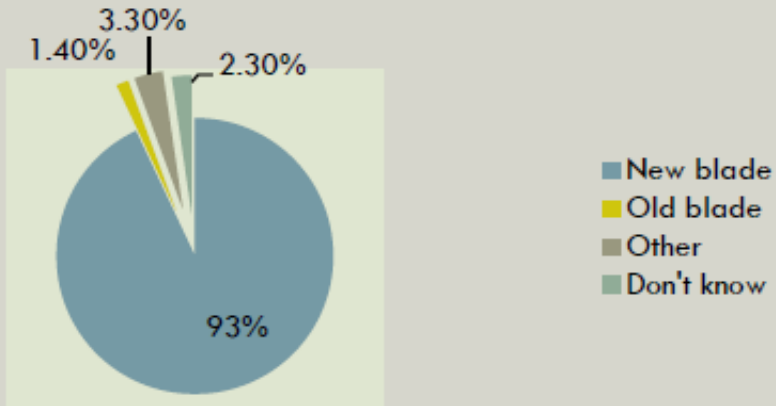


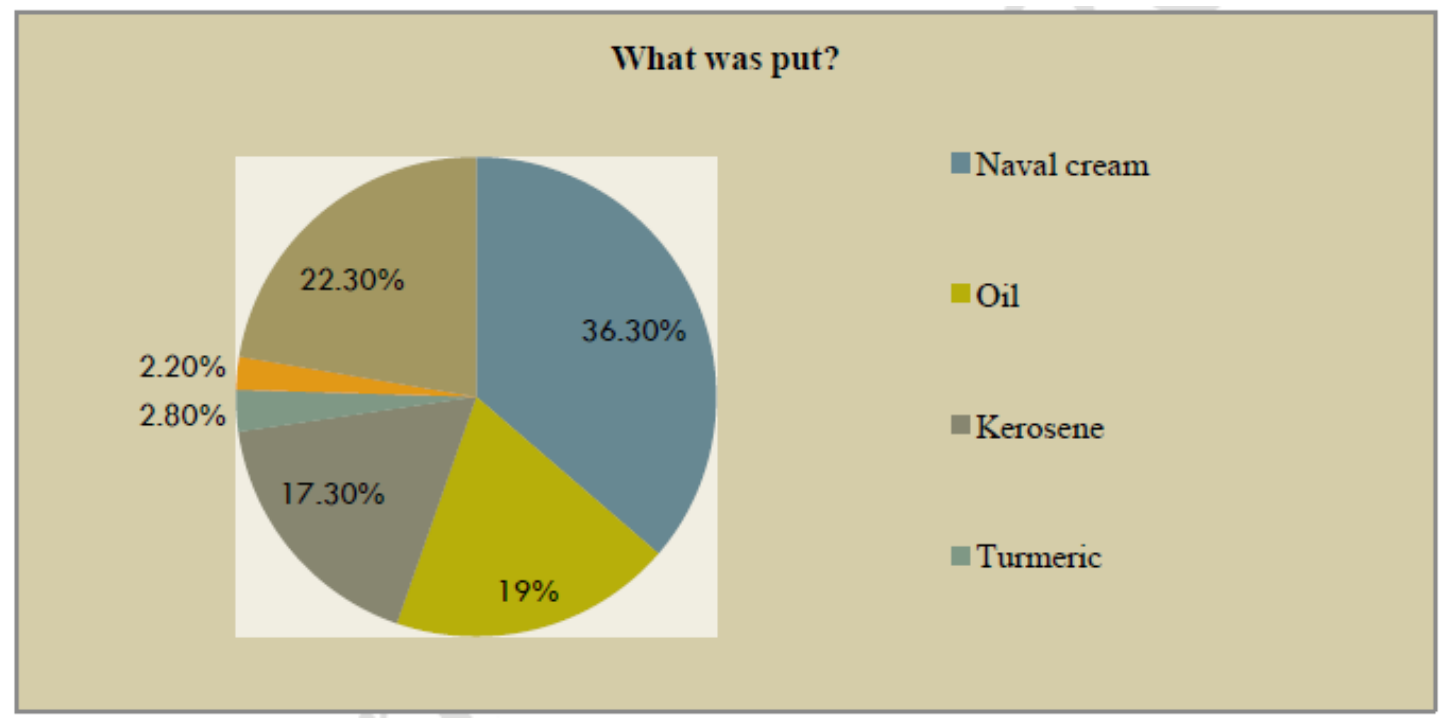

\section{Recommendations}

Based on the findings of behave mapping study, following recommendations are made.

- A very high proportion of women did not know about the obstetric and neonatal danger signs stressed the need for a plan to increase the awareness about such signs. There is an immediate need for strategic plan to increase the awareness of women about signs of obstetric complications. Health facility as well as out-reach clinics should be targeted for healtheducation on dangers signs of pregnancy, labour and after delivery to reach as many people as possible.

- Counselling women and husbands together by health worker may improve interpersonal communication among the couples. This may increase the service utilization as well as prevent harmful cultural practices.

- There is strong need to implement the community-based interventions to improve the new born care practices in community level and to reduce the high-risk new-born care practices like unsafe cord care, delayed breast feeding, early bathing, pre-lacteal feeding and discarding colostrum need through the community level health workers and volunteers.

- The study showed a strong influence of Mother In-Laws on the uptake of maternal and neonatal service utilization as well as household behaviour. Understanding their role is important if we are to design and target effective community-based health promotion interventions. Health promotion and educational interventions to improve the use of MNH should target women, husbands and family members, particularly mothers-in-law where they control access to family resources. Community mobilization and sensitization targeting women and men who provide finance for seeking care and are involved in decision making in the community is pre-requisite to improve $\mathrm{MNH}$ status in Kapilvastu district. Given the hierarchy of power and social position in Nepal, engaging mothers-in-law with establishing relationships of respect is one of the important steps required to change maternal health behaviour.

- While designing BCC as well as health promoting interventions, traditional views on pregnancy and motherhood as an important cultural factors influencing health seeking behaviour must not be overlooked. Allowing a family members (e.g. MILs, relatives, husbands, etc.) to accompany a woman during labour might be a possible intervention, to overcome the cultural need of family to be around and witness the delivery. It may also reduce the barrier of unfamiliar environment.

- Despite high coverage of ANC visit, very few women mentioned counselling on important aspects of MNH such as danger signs, birth preparedness, family planning, breastfeeding, newborn care, etc. Since the ANC clinic is the first contact with the women, it is necessary for the service providers to seize the opportunity and spend more 
time to give appropriate counselling, health education and discussion of plans for delivery. This will not only provide knowledge, but also build trust towards health facility.

- As the PNC behaviour significantly lags, efforts should be promoted to go for a PNC check-up within a week of birth (if the child is delivered in health facility) or immediately (if the child is delivered at home). In addition, a deliberate attempt is needed to deal with the culturally harmful practices prevailing during postnatal period.

- Recognizing the low awareness and use of family planning, there is a need to educate and motivate the couples and improve family planning services to achieve more effective and appropriate use of contraceptives. As almost all the women are breastfeeding their baby, the community's acceptance of breastfeeding presents an important opportunity in educating the population on LAM as one FP method.

- BCC intervention should specifically focus on prevailing malpractices such as keeping hair in the mouth of women to remove placenta and forcefully pressing lower abdomen during deliver; providing chilly soup of women during postnatal period; belief that providing nutritious food to pregnant women may increase fetal size which cause difficulty in delivery; applying kerosene oil in umbilical cord, prelacteal feeding; no handwashing before feeding newborn, etc.

- BCC package should be designed focusing on changing the adverse behaviour of women, mother-in-law, family members and Chamain. The information in the package should contained self-designed pamphlets, posters and visual cards containing simple messages in local languages (Awadhi). Culturally appropriate images should be designed based on community and subject expert feedback as well as a pilot field study. Rather than distributing such aids to women and communities, health workers, FCHVs and community mobilizers should use them while counselling and discussing with women and family members

\section{References}

[1]. ACHARYA, D. R., BELL, J. S., SIMKHADA, P., VAN TEIJLINGEN, E. R. \& REGMI, P. R. 2010. Research Women's autonomy in household decision-making: a demographic study in Nepal. Reproductive health, 7, 1-12.

[2]. DEPARTMENT OF HEALTH SERVICE 2014. Annual Report. Kathmandu, Nepal: Government of Nepal, Ministry of Health and Population, Department of Health Service.

[3]. GARG, P. \& GOGIA, S. 2009. Reducing neonatal mortality in developing countries: low-cost interventions are the key determinants. J Perinatol, 29, 74-75.

[4]. KABAKYENGA, J. K., ÖSTERGREN, P.-O., TURYAKIRA, E. \& PETTERSSON, K. O. 2012. Influence of birth preparedness, decision-making on location of birth and assistance by skilled birth attendants among women in south-western Uganda. PloS one, 7, e35747.

[5]. KAPHLE, S., HANCOCK, H. \& NEWMAN, L. A. 2013b. Childbirth traditions and cultural perceptions of safety in Nepal: Critical spaces to ensure the survival of mothers and newborns in remote mountain villages. Midwifery, 29, 1173-1181.

[6]. KERR, R. B., DAKISHONI, L., SHUMBA, L., MSACHI, R. \& CHIRWA, M. 2008. "We grandmothers know plenty": breastfeeding, complementary feeding and the multifaceted role of grandmothers in Malawi. Social Science \& Medicine, 66, 1095-1105.

[7]. KHAN, M. H., KHALIQUE, N., SIDDIQUI, A. R. \& AMIR, A. 2013. Impact of behavior change communication among pregnant women regarding neonatal care. The Indian Journal of Pediatrics, 80, 804-808.

[8]. MASVIE, H. 2006. The role of Tamang mothers-in-law in promoting breast feeding in Makwanpur District, Nepal. Midwifery, 22, 23-31.

[9]. MINISTRY OF HEALTH AND POPULATION-NEPAL 2007. National Medical Standard for Reproductive Health: Volume III Maternal and Neonatal Care. Kathmandu, Nepal: Family Health Division. 
Texila International Journal of Public Health

Volume 4, Issue 4, Dec 2016

[10]. MULLANY, B. C. 2006. Barriers to and attitudes towards promoting husbands' involvement in maternal health in Katmandu, Nepal. Social Science \& Medicine, 62, 2798-2809.

[11]. MULLANY, B. C., BECKER, S. \& HINDIN, M. 2007. The impact of including husbands in antenatal health education services on maternal health practices in urban Nepal: results from a randomized controlled trial. Health Education Research, 22, 166-176.

[12]. NAWAL, D. \& GOLI, S. 2013. Birth preparedness and its effect on place of delivery and postnatal check-ups in Nepal. PloS one, 8, e60957.

[13]. SIMKHADA, B., VAN TEIJLINGEN, E., PORTER, M. \& SIMKHADA, P. 2006a. Major problems and key issues in Maternal Health in Nepal. Kathmandu University Medical Journal, 4, 258263.

[14]. UNICEF 2009. The State of the World's Children 2009: Maternal and Newborn Health. Newyork, USA.

${ }^{\mathrm{i}}$ Chamain is a traditional birth attendance who is only allowed in the community to deliver the baby, ii Local made high calorie nutritious food of sugar, ginger,grains and dry foods

${ }^{\text {iii } V e r y ~ p o o r ~ m a r g i n a l i z e d ~ g r o u p ~ o f ~ p e o p l e ~ i n ~ N e p a l ~ c a l l e d ~ l o w e r ~ c a s t e ~ t o o . ~}$

iv the name-giving ceremony for the new born after 9 days

'Like Kangaroo mother care

${ }^{\mathrm{vi}}$ Higher caste people

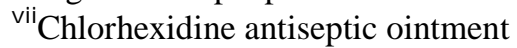

viii Cultural believe that kajal prevent the baby from evilsprit 